\title{
Binocular fusion: A test of the suppression theory'
}

Binocular fusion may be due to interocular inhibitory suppression, an hypothesis difficult to evaluate by phenomenal inspection. A test probe method (reaction time to a light pulse) was used to measure visual sensitivity during binocular rivalry and fusion. The absence of inhibitory effects during fusion fails to support the suppression theory of fusion.

Binocular fusion is said to occur when a single binocular percept is produced by similar targets striking corresponding parts of each retina. Although the term fusion adequately describes this unification of monocular stimulation, the mechanism underlying fusion is imperfectly understood. One widely held interpretation of fusion, the fusion theory, assumes that the monocular inputs contribute equally to the production of an emergent single percept. A less popular but enduring alternative interpretation, the suppression theory, asserts that fusion results from the suppression or inhibitory interaction of the monocular images, the single percept simply consisting of a mosaic of the dominant elements of each monocular view. Renewed interest in the suppression theory has recently developed, and arguments and data have been marshalled in its defense (e.g., Asher, 1953; Hochberg, 1964a, 1964b; Kaufman, 1964; Levelt, 1966).

Indirect support for the suppression theory is provided by the striking phenomenal suppression of dissimilar monocular targets found during binocular rivalry, in that the rivalry phenomenon demonstrates the existence of a powerful inhibitory mechanism which could exert the suppression effects demanded by the theory. Indeed, it is possible to argue that fused monocular targets are engaged in the oscillatory suppression characteristic of rivalry. Since fused targets must necessarily be quite similar, detection of alternating suppression through phenomenal inspection alone is quite difficult. In general, the absence of an unequivocal phenomenal indicator of suppression during fusion has largely precluded a direct test of the suppresion theory of fusion.

An indirect method of evaluating the suppression theory has been suggested by recent investigations of visual sensitivity during binocular rivalry (Fox, 1963, 1965). In these experiments on rivalry, the following method has been employed. A pair of monocular targets is aligned so as to generate active rivalry. An experienced observer continuously reports, by means of a switch, the dominance and suppression phases of the targets. One target is arbitrarily designated as the suppression target. During the suppression and the dominance periods of that target a test stimulus is superimposed upon the target. The observer makes an appropriate response to the test stimulus (e.g., he may indicate detection of the stimulus or may give a reaction time response). The suppression phase of the target can be regarded as the independent variable while the nonsuppression phase furnishes the control condition. The response to the test stimulus is the dependent variable. Differences in response between suppression and nonsuppression phases provide a measure of the effect of suppression upon test stimuli. Through appropriate combinations of test stimuli and indicator responses, inferences can be made about the operations of the rivalry suppression mechanism.

This general paradigm, which may be called the test probe method, is not unique. Analogous procedures have been employed in the study of perceptual correlates of phasic EEG activity (Dustman, 1963) and in studies of backward masking (Raab, 1963).

Application of the method to binocular rivalry has revealed two facts of special relevance to the suppression theory of fusion. (1) The suppression state in rivalry acts to inhibit or attenuate test stimuli; i.e., the increment threshold is raised. (2) The inhibitory suppression state operates nonselectively; i.e., many classes of test stimuli (e.g., form, movement, color) are attenuated, independently of the similarity between the suppression target and the test stimulus. These results are congruent with certain assumptions of the suppression theory of fusion.

The logic of the test probe method can be extended to the binocular fusion situation without difficulty. The independent variable is the condition where two monocular targets are fused. Control treatments are provided by presentation of the targets under certain monocular and nonfusion binocular conditions. If a mechanism similar to rivalry suppression is operative during fusion, then an inhibitory effect of fusion upon a test stimulus is to be anticipated.

The suppression theory of fusion clearly requires inhibition of one monocular target, but the temporal pattern of such inhibition has not yet been specified. Three alternative hypotheses can be entertained at present: (1) The fused targets may be engaged in alternating suppression, following the same pattern observed for binocular rivalry. On a priori grounds this is perhaps the most reasonable and parsimonious assumption. (2) The targets may interact to produce continuous suppression of one target; i.e., there is not interocular alternation of suppression. (3) The targets may be engaged in alternating suppression, but following a sequence independent of the pattern observed during 
rivalry. These three hypotheses are tested in the two experiments described in this paper. The first experiment was designed specifically to test hypothesis 1 , while the second experiment bears more directly upon hypotheses 2 and 3 .

\section{EXPERIMENT I}

\section{Subjects}

Two Ss with normal vision and experienced in observing rivalry were employed. LS was left-eye dominant and MG was right-eye dominant, as measured by the Miles funnel test.

\section{Apparatus}

A Wheatstone-type stereoscope, assembled from optic bench components, was used to present the left and right eye field targets. Each field consisted of a white $7^{\circ}$ square, transilluminated by an incandescent source. The luminance of the white area of each field was $55 \mathrm{ft}$.-L. Along each vertical border of each square two 17' black stripes, equally spaced, were located. These stripes served to enhance fusion of the squares. The rivalry targets, each a $1^{\circ} 20^{\prime}$ square formed from 17' black lines, were located in the center of each square. The 57' interior of the left eye target was green, while the corresponding right eye target area was red. The hues were produced by gelatin theatrical filters. Black 3 ' stripes, equally spaced, covered the chromatic area of each target; for the green left field target, the stripes were oriented $45^{\circ}$ to the left of vertical; for the red square, $45^{\circ}$ to the right. During rivalry the black borders of the targets remained fused while the colors and diagonal lines within the borders engaged in active rivalry. Under some conditions a fusion stimulus, consisting of a $33^{\prime}$ black square, was added to each field, one fusion square being located on the vertical midline above each rivalry target; the distance between the top border of the rivalry targetand the bottom border of the fusion square was $1^{\circ} 20^{\prime}$. The test stimulus was a light flash superimposed optically either in the center of the right field fusion square or in the center of the right field rivalry target. The flash was generated by the lamp-driver and timing units of a 3-field tachistoscope (Scientific Prototype Mfg. Corp., Model H). Flash duration was .1 sec.; luminance, $32 \mathrm{ft}$.-L.; diameter $11^{\prime}$. The observers viewed the fields through $2 \mathrm{~mm}$ artificial pupils.

To signal the dominance and suppression phases of the rivalry targets a lever-type switch was' depressed and released. The reaction time response to the test flash was release of a microswitch mounted on the key used to report the rivalry alternatives. Extensive preliminary tests had shown that operation of the rivalry observation key did not interfere with the reaction time response for experienced subjects. Duration of reaction time was measured by a Hunter Klockounter, the counter, connected to a tachistoscope timer through a transistor gate, started simultaneously with onset of the test flash.

To initiate the events culminating in presentation of a test flash the experimenter temporarily connected the rivalry observation key to the set of timing-switch units. Whenever $\mathbf{S}$ moved the key, indicating the start of the appropriate phase (either suppression or nonsuppression), the timing units were activated and these, in turn, triggered the test flash. An operations recorder recorded the duration of the rivalry fluctuations and the intratrial events.

A Spectra Photo Brightness Meter was used periodically to check luminance values. A digital counter was used to check the timing units.

\section{Design and Procedure.}

The basic task of the Ss was composed of two coextensive actions: (1) signalling the dominance and suppression phases of the rivalry targets continuously via the rivalry observation key, and (2) reacting to the onset of the test flash.

The rivalry observations and the reaction trials were performed under five conditions of test flash presentation. These were (1) Binocular rivalry: the test flash was located in the center of the right field rivalry target. (2) Binocular fusion: the black fusion squares located above each rivalry target were carefully aligned to produce fusion without stereopsis; the test flash was placed in the center of the right field fusion square. (3) Monocular black: the test flash was centered in the right field fusion square; the left field square was absent. (4) Binocular suppression; the left field fusion square was present and the right field fusion square was absent; the test flash was positioned in the area assigned to the right field square. (5) Binocular white: both fusion squares were absent and the test flash was positioned in the area assigned to the right field fusion square.

The five conditions were administered in accord with a randomized block design: under each condition a total of 100 suppression and 100 nonsuppression reaction times were obtained from each $\mathrm{S}$. In a single session, 50 reactions were taken; within each condition, five suppression and five nonsuppression trials were administered following predetermined random schedules.

The procedures employed for each trial involved the following steps. Once fusion of the rivalry squares was obtained, the S observed two to five cycles of rivalry. In one of these cycles the test flash was presented. The cycle number and the appropriate phase (suppression or nonsuppression) for presentation of the test flash was governed by a predetermined quasi-random schedule. The onset of the flash occurred .6 sec. after the $\mathrm{S}$ signaled the beginning of the phase; reaction to the flash terminated the trial (the intensity of the flash was sufficient to elicit a reaction under all conditions). After making the response the $\mathbf{S}$ gave a confirmatory verbal report indicating the rivalry phase actually present during the onset of the flash. This procedure provided a control for observation errors and spontaneous changes in the rivalry state. Trials were accepted only if the confirmatory report agreed with the state 
Table 1. Reaction time (msec,) for suppression and nonsuppression phases under five stimulus conditions.

\begin{tabular}{|c|c|c|c|c|c|c|c|c|c|c|c|}
\hline \multirow[b]{3}{*}{ Subject } & \multirow[b]{3}{*}{ Phase } & \multicolumn{10}{|c|}{ Stimulus Condition } \\
\hline & & \multicolumn{2}{|c|}{ Bin. Rivalry } & \multicolumn{2}{|c|}{ Bin. Fusion } & \multicolumn{2}{|c|}{ Mon. Black } & \multicolumn{2}{|c|}{ Bin. White } & \multicolumn{2}{|c|}{ Bin. Supp. } \\
\hline & & Meon & S.D. & Mean & S.D. & Mean & S.D. & Mean & S.D. & Mean & S.D. \\
\hline \multirow{3}{*}{ LS } & Supp. & 239.7 & 19.8 & 250.0 & 16.6 & 246.6 & 16.0 & 254.0 & 24.4 & 270.7 & 20.4 \\
\hline & Nons. & 232.7 & 19.4 & 246.0 & 19.6 & 247.0 & 17.4 & 258.0 & 27.0 & 272.0 & 22.4 \\
\hline & & $p<.02$ & & & & & & & & & \\
\hline \multirow[t]{2}{*}{ MG } & $\begin{array}{l}\text { Supp. } \\
\text { Nons }\end{array}$ & $\begin{array}{l}335.0 \\
318.7\end{array}$ & $\begin{array}{l}39.7 \\
35.2\end{array}$ & $\begin{array}{l}374.6 \\
362\end{array}$ & $\begin{array}{l}52.7 \\
36.6\end{array}$ & $\begin{array}{l}371.6 \\
3578\end{array}$ & 42.7 & $\begin{array}{l}377.1 \\
370.9\end{array}$ & 47.7 & 394.1 & $\begin{array}{l}47.8 \\
44.2\end{array}$ \\
\hline & & $p<.01$ & & 302.1 & & 837.8 & & 30.9 & & 308.0 & \\
\hline LS & Comb. & & & 248.0 & & 246.8 & & 256.0 & & $271.4^{*}$ & \\
\hline$M G$ & Comb. & & & 368.8 & & 364.7 & & 374.0 & & $391.4^{*}$ & \\
\hline
\end{tabular}

* This value differs significantly $(p<.05)$ from the remaining four for each $S$.

indicated by the rivalry observation key. The Ss were not informed about the acceptance or rejection of a trial. Although the absolute number of disconfirmed trials was quite small, confirmatory reports were taken throughout the experiment.

Prior to formal data collection, each S received about 150 practice trials. The Ss were trained to assume a stringent criterion for a rivalry change, and to report a change only when complete dominance or suppression of the targets had occurred. For both Ss the durations of suppression and nonsuppression phases were approximately equal and on the order of 3-4 sec.

Subject MG was run without a ready signal. For LS, an auditory ready signal (1000 cycle tone, .1 sec. duration) was employed together with a constant .5 sec. foreperiod. To control for the constant foreperiod, 160 blank trials were distributed randomly throughout the schedule of trials for LS.

\section{Results}

In Table 1 the reaction time means and standard deviations for suppression and nonsuppression phases under all conditions are given. The faster reactions displayed by LS undoubtedly are due to the ready signal. The overall faster reactions for both Ss under the rivalry condition are the result of the foveal position of the test flash for that condition.

The main analysis of these data involved testing the significance of the differences between suppression and nonsuppression reaction times for the five conditions in order to evaluate hypothesis 1 (i.e., that the fusion squares engage in an alternating suppression which follows the pattern of rivalry alternation). The Wilcoxon paired replicates test and an $F$ test were applied to each difference in mean reaction time. By both tests and for both $\mathrm{Ss}$, mean suppression reaction time is significantly greater than the nonsuppression time for the rivalry condition; this outcome is consistent with previous work. However, for the four nonrivalry conditions the differences do not approach acceptable significance levels. Failure to obtain a suppression-nonsuppression difference under the fusion condition provides no support for hypothesis 1 .

If either hypothesis 2 or 3 is correct, reaction times under fusion should be significantly greater than under the monocular conditions. A separate analysis of the four nonrivalry conditions, with suppression and nonsuppression reactions combined, indicates for both Ss that the fusion, monocular black, and binocular white means do not differ significantly (as assessed by the Newman-Keuls multiple comparison procedure, with $p=.05)$. The similarity of the fusion mean to monocular conditions is not consistent with either hypothesis 2 or 3 . Yet mean reaction time for the binocular suppression condition is significantly greater than any of the other three conditions for both Ss. This increase suggests that inhibitory suppression effects may be present for certain classes of binocular targets other than ones engaged in active rivalry; in this case, when a figure is paired with the ground of the second monocular field.

\section{EXPERIMENT II}

In this experiment the hypotheses of permanent suppression and of oscillatory suppression during fusion were investigated for foveally located targets.

\section{Subjects}

Four Ss were used: LS and MG (from Experiment I) and $\mathrm{EW}$ and $\mathrm{CY}$, who were naive regarding the purpose but experienced in psychophysical observation. Both of these latter Ss were left-eye dominant.

\section{Apparafus}

The apparatus was similar to that used in the first experiment. The principal difference consisted of moving the $33^{\prime}$ black fusion targets for the four nonrivalry conditions to the fovea and eliminating the colored rivalry squares. To maintain fixation the targets were placed at the center of a $1^{\circ} 50^{\prime}$ square formed by four $16^{\prime}$ circles.

Further, a pair of targets was constructed for a new, fifth condition called permanent suppression. For this condition the right eye field target was simply the black fusion square, foveally located. The left eye field target was a replica of the left field rivalry target used in Experiment I but reduced in size to match the fusion square. When the left and right field targets were fused the left field rivalry target completely suppressed the fusion square for continuous observation periods of at least $60 \mathrm{sec}$, after which time active rivalry between 
Table 2. Reaction time (msec.) under five stimulus conditions.

\begin{tabular}{|c|c|c|c|c|c|c|c|c|c|c|}
\hline \multirow[b]{3}{*}{ Subject } & \multicolumn{10}{|c|}{ Stimulus Condition } \\
\hline & \multicolumn{2}{|c|}{ Bin. Fusion } & \multicolumn{2}{|c|}{ Mon. Block } & \multicolumn{2}{|c|}{ Bin. White } & \multicolumn{2}{|c|}{ Bin. Supp. } & \multicolumn{2}{|c|}{ Perm. Supp. } \\
\hline & Mean & S.D. & Mean & S.D. & Mean & S.D. & Mean & S.D. & Mean & S.D. \\
\hline$E W$ & 228.3 & 20.9 & 220.6 & 17.9 & 223.5 & 18.1 & 232.1 & 20.7 & 221.4 & 13.5 \\
\hline $\mathrm{CY}$ & 250.7 & 11.4 & 261.0 & 23.9 & 259.6 & 19.4 & 265.0 & 26.9 & 254.8 & 13.9 \\
\hline LS & 234.4 & 12.7 & 236.6 & 12.0 & 242.2 & 15.3 & $269.6^{*}$ & 15.6 & $258.6^{*}$ & 18.6 \\
\hline MG & 298.9 & 19.6 & 291.1 & 18.4 & 303.2 & 9.1 & 301.4 & 11.1 & 298.1 & 15.8 \\
\hline
\end{tabular}

* These values differ significantly $(p<.05)$ from the remaining three for this $S$.

the targets began. For the brief periods of observation (e.g., 15 sec.) constituting a trial in this experiment, suppression of the fusion square by the rivalry target was absolute. Thus, the permanent suppression condition provided a case where figure suppresses figure but rivalry is not operative.

\section{Design and Procedure.}

The five stimulus conditions were administered following a randomized block desigm, with 100 reaction times taken from each $\mathrm{S}$ under each of the five conditions. In a single session 50 trials were run. An auditory ready signal with a constant $.5 \mathrm{sec}$. foreperiod was used with all Ss; 80 catch trials were dispersed randomly throughout the schedule of presentation. All Ss received at least 150 practice trials before formal data were collected.

\section{Resulis}

Table 2 contains the reaction time means and standard deviations for each $S$ under the five conditions. For Ss MG, EW, and CY the differences between the means are not significant between any of the five conditions. For LS the differences between the fusion, binocular white, and monocular black conditions are not significant. The similarity of the fusion condition to control conditions (monocular black and binocular white) indicates that inhibitory suppression effects are not operating during fusion. Since fusion reaction time is definitely not increased relative to control conditions, neither hypothesis 2 nor 3 is supported.

Evidence for a suppression effect produced by dissimilar binocular stimulation is provided by the elevated reaction times given by LSunder the binocular suppression and permanent suppression conditions. Both of these means are significantly greater than the other three conditions, as assessed by the Newman-Keuls procedure with $\mathrm{p}=.05$. Although a similar suppression effect was found in Experiment I for both LS and MG, only LS displayed the effect in the second experiment. The reason for this discrepancy is not clear; however, the presence or absence of such suppression effects is not critical for the main argument of the suppression theory.

None of the three alternative hypotheses concerming the operation of suppression was supported by the results of Experiments I and II. It may be possible to derive other hypotheses, but the pattern of means obtained in Experiment II, together with the eye dominance of the Ss, does not seem consistent with any reasonable derivations from the suppression theory. The major conclusion to be drawn from the results is that an inhibitory suppression process does not occur during binocular fusion; therefore, the suppression theory of fusion is not supported.

Yet it should be added that current formulations of the suppression theory employ a minimum number of strong but loosely articulated assumptions; for example, suppression of one target must occur whenever phenomenal fusion is present. There is no reason why such assumptions cannot be modified to yield a more workable version of the theory. One possible modification might be an assumption which relates the likelihood of suppression to the complexity of the fused monocular targets, i.e., perhaps suppression occurs only when the presence of many contours in each target generates alignment problems for the eyes. If such modifications of the theory are proposed, it should be possible to test them by the general paradigm described in this report.

\section{References}

Asher, H. Suppression theory of binocular vision. Brit. J. Ophthalm., $1853,37,37-49$

Dustman, R. E. Phase of alpha brain waves, reaction time, and visually evoked potentials. Unpublished doctoral dissertation, University of Utah, 1963.

Fox, R. An analysis of the suppression mechanism in binocular rivalry. Unpublished doctoral dissertation, University of Cincinnati, 1963.

Fox, R. The suppression mechanism in binocular rivalry: movement detection. Paper read at Psychonomic Society, Chicago, 1965.

Hochberg, J. Depth perception loss with local monocular suppression: a problem in the explanation of stereopsis. Science, 1964a, $145,1334$.

Hochberg, $\mathbf{J}$. The suppression and imitation of binocular stereopsis. Paper read at Psychonomic Society, Niagara Falls, 1964 b.

Kaufman, L. On the nature of binocular disparity. Amer. J. Psychol., $1964,77,393-402$.

Levelt, W. J. M. Some demonstrations of the complementary functioning of the eyes. Percept. \& Psychophys., 1966, 1, 39-40. Raab, D. H. Backward masking. Psychol. Bull., 1963, 60, 118-129. Note

1. This research was supported by grant MH0834 from the U, S Public Health Service.

(Accepted for publication August 15, 1966.) 